\title{
Critical Phenomena in the Temperature-Pressure-Crowding Phase Diagram of a Protein
}

\author{
Andrei G. Gasic $\odot,{ }^{1,2,{ }^{*}}$ Mayank M. Boob $\odot,{ }^{3, *}$ Maxim B. Prigozhin $\odot,{ }^{4, \dagger}$ Dirar Homouz, ${ }^{1,2,5}$ \\ Caleb M. Daugherty, ${ }^{1,2}$ Martin Gruebele $\odot,{ }^{3,4,6, \$}$ and Margaret S. Cheung $\oplus^{1,2, \S}$ \\ ${ }^{1}$ Department of Physics, University of Houston, Houston, Texas 77204, USA \\ ${ }^{2}$ Center for Theoretical Biological Physics, Rice University, Texas 77005, USA \\ ${ }^{3}$ Center for Biophysics and Quantitative Biology, University of Illinois at Urbana-Champaign, \\ Champaign, Illinois 61801, USA \\ ${ }^{4}$ Department of Chemistry, University of Illinois at Urbana-Champaign, Champaign, Illinois 61801, USA \\ ${ }^{5}$ Department of Physics, Khalifa University of Science and Technology, \\ P.O. Box 127788, Abu Dhabi, United Arab Emirates \\ ${ }^{6}$ Department of Physics and Beckman Institute for Advanced Science and Technology, \\ University of Illinois at Urbana-Champaign, Champaign, Illinois 61801, USA
}

(Received 11 June 2019; revised manuscript received 21 September 2019; published 18 November 2019)

Inside the cell, proteins fold and perform complex functions through global structural rearrangements. For proper function, they need to be at the brink of instability to be susceptible to small environmental fluctuations yet stable enough to maintain structural integrity. These apparently conflicting properties are exhibited by systems near a critical point, where distinct phases merge. This concept goes beyond previous studies that propose proteins have a well-defined folded and unfolded phase boundary in the pressuretemperature plane. Here, by modeling the protein phosphoglycerate kinase (PGK) on the temperature $(T)$, pressure $(P)$, and crowding volume-fraction $(\phi)$ phase diagram, we demonstrate a critical transition where phases merge, and PGK exhibits large structural fluctuations. Above the critical temperature $\left(T_{c}\right)$, the difference between the intermediate and unfolded phases disappears. When $\phi$ increases, the $T_{c}$ moves to a lower $T$. With experiments mapping the $T-P-\phi$ space, we verify the calculations and reveal a critical point at $305 \mathrm{~K}$ and $170 \mathrm{MPa}$ that moves to a lower $T$ as $\phi$ increases. Crowding shifts PGK closer to a critical line in its natural parameter space, where large conformational changes can occur without costly free-energy barriers. Specific structures are proposed for each phase based on the simulation.

DOI: 10.1103/PhysRevX.9.041035

\section{INTRODUCTION}

Complex processes in nature often arise at a transition between order and disorder [1-4]. In proteins, this complexity arises from an almost perfect compensation of entropy by enthalpy; molecular interactions that create their structural integrity are on the same scale as thermal fluctuations from the environment. The resulting marginal stability of proteins suggests that they could behave like fluids near a critical point [5]. That is, when subject to small

\footnotetext{
*These authors contributed equally to this work.

${ }^{\dagger}$ Present address: Department of Physics, James H. Clark Center, Stanford University, Stanford, CA 94305, USA.

‡mgruebel@illinois.edu

§mscheung@uh.edu

Published by the American Physical Society under the terms of the Creative Commons Attribution 4.0 International license. Further distribution of this work must maintain attribution to the author(s) and the published article's title, journal citation, and DOI.
}

Subject Areas: Biological Physics, Soft Matter, Statistical Physics perturbations, their structures fluctuate considerably without overcoming a large activation barrier.

The concept of first-order and critical phase transitions does not strictly apply to nano-objects such as proteins; nevertheless, it is a useful one to classify folding transitions. For example, the folding of some small model proteins is described as an abrupt, cooperative transition between their folded and unfolded phases (the belowcritical point scenario) or for other proteins as a gradual barrierless "downhill" transition (the above-critical point scenario) [6]. Even though critical behavior of proteins has been previously suggested [7-9], a critical point where one of these abrupt transitions simply disappears at $T_{c}$ and $P_{c}$ has not been demonstrated. In larger proteins, such as phosphoglycerate kinase (PGK) (Ref. [10], Sec. S1), there is the added complexity that different parts or "domains" of such a large protein are more likely to undergo separate order-disorder events [11], delicately poised between folded and partially unfolded structures, to carry out their functions [12].

Proteins must fold and function while crowded by surrounding macromolecules [13], which perturb their 

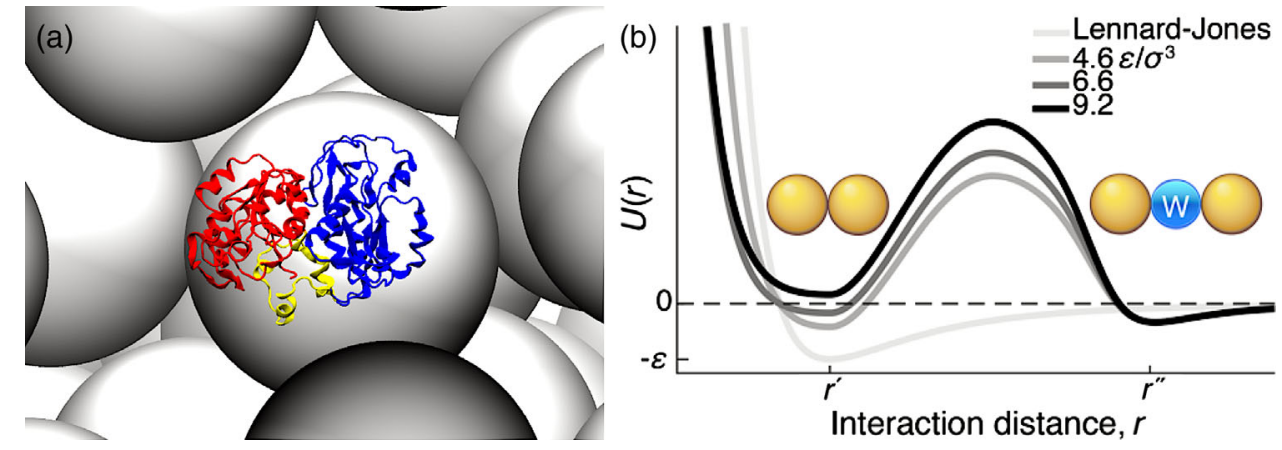

FIG. 1. PGK surrounded by crowders and the desolvation potential between residues. (a) A snapshot from the coarse-grained molecular simulation of PGK's spherical compact state (SPH) surrounded by crowders (gray) at the volume fraction of $40 \%$. The N and $\mathrm{C}$ domain and hinge are in red, blue, and yellow, respectively. (b) The pressure-dependent desolvation potential at $\sigma^{3} P / \epsilon_{0}=4.6,6.6$, and $9.2\left(\epsilon_{0} / \sigma^{3} \approx 76 \mathrm{MPa}\right)$ contains a desolvation barrier with a width $\left(\left|r^{\prime}-r^{\prime \prime}\right|\right)$ the size of a water molecule (blue). This barrier incorporates the entropic cost of expelling a solvent molecule between two residues (gold). The Lennard-Jones potential is plotted in light gray for comparison.

structure at physiological conditions in the cell. In living cells, the volume exclusion from macromolecules [14], which places shape and size (or covolume) [15,16] constraints on the conformational space [Fig. 1(a)], complicates protein folding and dynamics [17]. How the competing properties of a protein arise-being both stable yet dynamically sensitive to its environment-is virtually unknown. Herein, we show that the crowded environment provides a unique solution by placing PGK closer to a critical regime.

We use pressure $P$, temperature $T$, and the crowderexcluded volume fraction $\phi$ to map PGK's folding energy landscape $[12,18]$ and its critical regime on the $T-P-\phi$ phase diagram. Temperature can induce heat unfolding by favoring states of high conformational entropy or cold denaturing by favoring reduced solvent entropy when hydrating core amino acids in the protein $[19,20]$. Since folded proteins contain heterogeneously distributed small, dry cavities due to imperfect packing of their quasifractal topology [21-23], high pressure also induces unfolding by introducing water molecules (as small granular particles) into the cavities in protein structures. This process reduces the overall solventaccessible volume of the unfolded protein [24]. In the presence of high crowding (large excluded volume fraction $\phi)$, compact desolvated (crystal) states are favored over less compact solvated (unfolded) states [12].

To investigate the opposing influence of macromolecular volume exclusion and solvation water on protein conformation, we utilize a minimalist protein model (see Appendix A and Ref. [10], Sec. S2.2) that incorporates the free-energy cost of expelling a water molecule between a pair of amino-acid residues in a contact termed the desolvation potential [Fig. 1(b)] [25]. This potential has a barrier that separates two minima, which represent a native contact and a water-mediated contact. As pressure increases, the desolvation barrier increases, and the free-energy gap between the two minima tilts to favor the water-mediated contact, which leads to an unfolding of a protein, which captures the main feature of pressure denaturation. Despite the model's simplicity, without all the detailed chemistry in a residue [26], this desolvation model predicts a folding mechanism based on water expulsion from the hydrophobic core, which is observed by all-atomistic molecular dynamics [27] and validated by experiments in which the volume or polarity of amino acids is changed by mutation [28]. We previously employed a similar model without a desolvation potential to investigate compact conformations of PGK induced by macromolecular crowding [12]. Now, by studying the competition of temperature, pressure, and crowding on the energy landscape, we observe that a costly barrier between two specific phases disappears, along a critical line on top of the isochore surface. As such, the current investigation demonstrates a richer ensemble of PGK states (Sec. II A) than our previous study [12].

To test our computational model experimentally, we observe by fluorescence the structural transitions of PGK and construct the experimental $T-P-\phi$ phase diagram (Sec. II B). These experiments verify our prediction of the existence of a critical point and that $T_{c}$ moves to a lower temperature $T$ as the crowding volume fraction $\phi$ increases. Using scaling arguments from polymer physics, we derive a critical line $T_{c}(\phi)$ and present a unified phase diagram (Sec. II C) to investigate the underlying physical origin of such a transition. We also discuss the consequence of the existence of criticality in PGK's phase diagram and the possible relation to its function. Even though the observed critical point is most relevant to physiological conditions for extremophile organisms [30], its influence may extend over a broader range of the phase diagram. The current investigation adds complexity to the typical "structurefunction" relation for proteins to include the novel role of the environment in this relationship and contributes toward developing universal thermodynamic principles of protein folding in living cells. 


\section{RESULTS AND DISCUSSION}

\section{A. Computational $T-P-\phi$ phase diagram of PGK}

We investigate the conformations of PGK, a large, 415amino-acid, two-domain protein (see Ref. [10], Sec. S1, for more information on PGK), in an environment containing Ficoll 70, which acts as a crowding agent to mimic cell-like excluded volume. As it is known to be inert to proteins and behaves as a semirigid sphere [31,32], Ficoll 70 is computationally modeled as a hard sphere. From prior Förster resonance energy transfer (FRET) experiments and molecular simulations, we observe several PGK conformations that denote a phase diagram in the $\phi-T$ plane [12]. It includes four states: C (crystal structure), CC (collapsed crystal), SPH (spherically compact state), and U (unfolded structures). In the $\mathrm{C}$ state, there is a linker that separates the $\mathrm{N}$-terminal and C-terminal domains, resembling an open "pacman." The CC state is a closed pacman. The SPH state involves a twisting of one of the domains with respect to the other and becomes more spherical than the CC state. A complete description of the structures of these states is in Supplemental Material [10], Sec. S3.

By changing hydrostatic pressure $P$ and the volume fraction of crowders $\phi$ at several temperatures $T$, we identify two new states on the $\phi-P$ isothermal phase plane (Fig. 2): I (folding intermediate) and SU (swollen compact unfolded structure). The criteria to define the six distinctive conformations are in Table S3.1 [10]. The I state is an ensemble of structures containing one folded domain (C terminus) and one unfolded domain ( $\mathrm{N}$ terminus), indicating it to be the least stable domain on its own. SU is completely denatured but exhibits many watermediated contacts [Fig. 2(b)]. Thus, the SU state is structurally more compact than the U state.

The microscopic mechanism of the pressure-induced unfolding of PGK depends on $T$ and $\phi$. Figure 2 shows
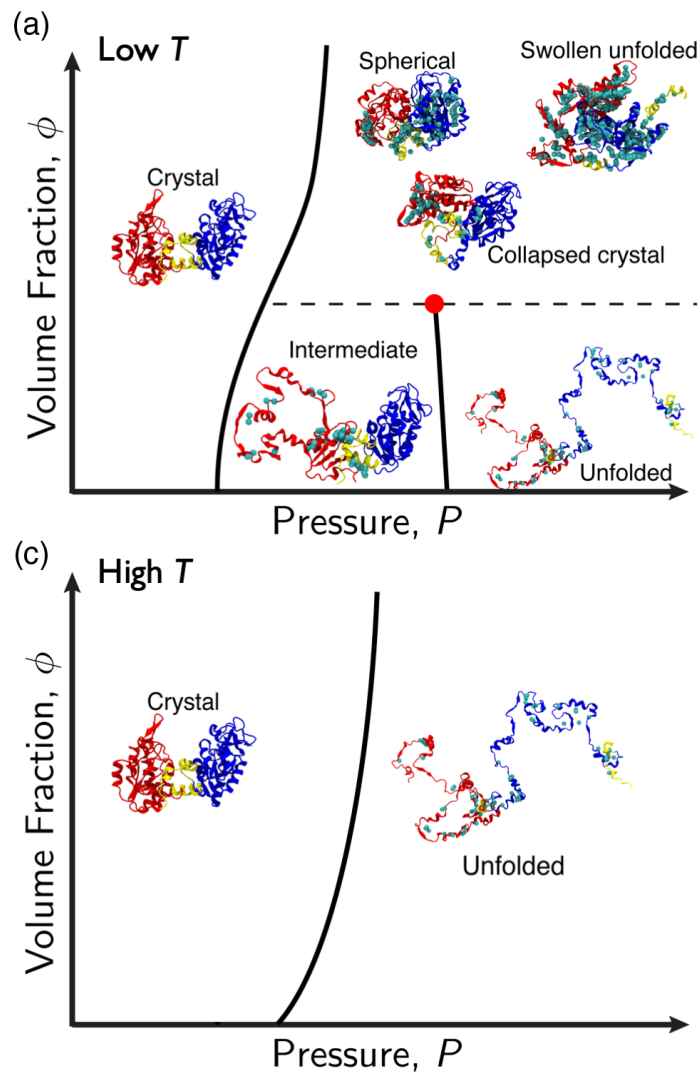

(b)

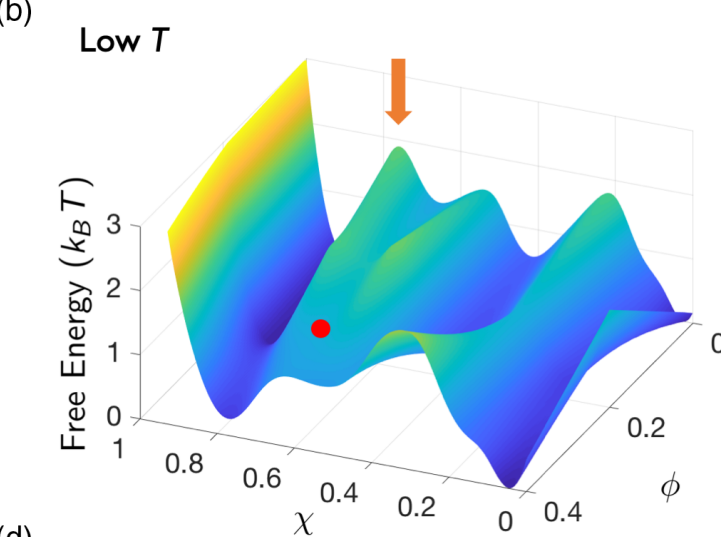

High $T$

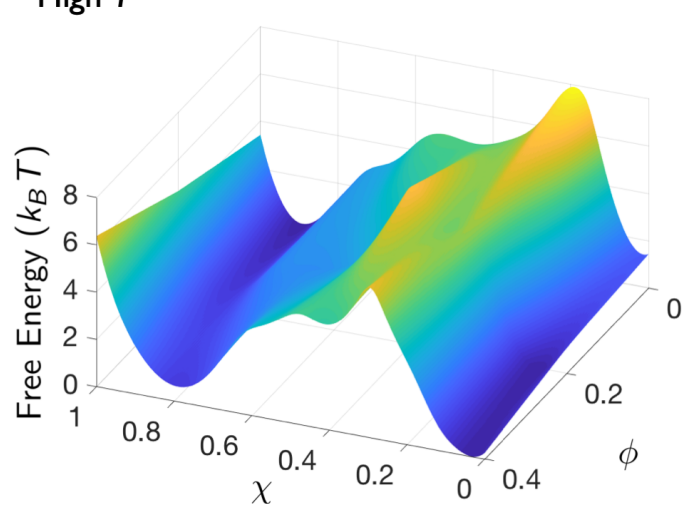

FIG. 2. Solvation and crowding give rise to an intricate phase diagram of PGK. (a),(c) Schematics of PGK's behavior in the crowding volume-fraction-pressure $(\phi-P)$ phase plane and (b),(d) corresponding free energy with respect to the overlap $\chi$ and crowding volume fraction $\phi$ at the folding pressure at low (a),(b) and high (c),(d) temperatures. Solid lines represent the division between distinct configurational phases that are separated by a free-energy barrier from simulations at $\phi=0,0.2$, and 0.4 and pressures from $\sigma^{3} P / \epsilon_{0}=$ $10^{3}$ to $13\left(\epsilon_{0} / \sigma^{3} \approx 76 \mathrm{MPa}\right)$. The dashed line (a) represents a continuous transition along $\phi$, and red dots (a),(b) represent an approximate position of the critical points. The orange arrow (b) marks the peak of the barrier that diminishes until it disappears after the critical point. Collapsed crystal, spherical, and swollen unfolded states are indistinguishable in terms of free energy. These configurations are reconstructed from coarse-grained models to all-atomistic protein models for illustration purposes using SCAAL (side chain- $C_{\alpha}$ to all-atom method) [29]. The $\mathrm{N}$ and $\mathrm{C}$ domains and hinge are in red, blue, and yellow, respectively. A cyan sphere is inserted in between residues to show water-mediated contacts. 
the $P-\phi$ phase plane at low $T$ in Fig. 2(a) and high $T$ in Fig. 2(c). At sufficiently low $T$ and $\phi=0$ (no crowders), the unfolding of PGK is a multistate transition between crystal state C [Figs. 2(a) and 2(b)] and unfolded state U via an intermediate state I. We capture the folding process using the overlap parameter $\chi$ :

$$
\chi \equiv 1-\frac{1}{N^{2}-5 N+6} \sum_{i=1}^{N-3} \sum_{j=i+3}^{N} \Theta\left(1.2 r_{i j}^{0}-r_{i j}\right),
$$

where $N$ is the number of residues $(=415), \Theta$ is the Heaviside step function, $r_{i j}$ is the distance between the residues $i$ and $j$ for a given conformation, and $r_{i j}^{0}$ is that corresponding distance in the crystal structure. It characterizes similarity to the crystal structure, $\mathrm{C}$ state. $\chi$ ranges from 0 to 1 , where 0 represents the $\mathrm{C}$ state. In Fig. 2(b), at $\phi=0$, the $\langle\chi\rangle$ of the I state, $\chi_{I}$, approximately equals 0.35 , and the $\langle\chi\rangle$ of the U state, $\chi_{U}$, approximately equals 0.9 . The state I is a consequence of the heterogeneous distribution of cavities, which causes uneven pressure denaturation as the $\mathrm{N}$ domain unfolds, but the $\mathrm{C}$ domain remains intact. Since the total cavity volume of the N-terminal domain (approximately equal to $171 \AA^{3}$ ) is about a third larger than that of the C-terminal domain (approximately equal to $132 \AA^{3}$ ), the former is more vulnerable to high pressure. Moreover, two antiparallel $\beta$ strands $m$ and $n$ of the Nterminal domain are totally exposed to the solvent (see Ref. [10], Sec. S5 and Fig. S1.1). Under high pressure, they act as a channel for water to fill the N-terminal domain's cavities.

At sufficiently high $\phi$ and low $T$ [Fig. 2(a), above the red critical point], there is only a single transition due to pressure between a crystal state and several compact states (SPH, CC, and $\mathrm{SU}$ ) without the I state. The transition from $\mathrm{C}$ to SPH or $\mathrm{CC}$ states involves domain rearrangement where the linker "cracks" [11] and forms a disordered hinge. Furthermore, high pressure competing with crowding gives rise to a compact unfolded conformation where up to half of the contacts become swollen with water that forms a "wet interface" (SU). As the limited void formed by the density fluctuations of crowders inhibits extended conformations [33], the $U$ state is unfavored due to macromolecular crowding [29]. The protein needs only to reduce its volume slightly as it expels water molecules out of this wet core to return to the SPH or CC states from the SU state. Effectively, no barriers exist between the $\mathrm{SPH}, \mathrm{CC}$, and $\mathrm{SU}$ states, which are located in the same region of the phase diagram [see Figs. 2(a) and 2(b) at $\chi=0.4$ to 0.8 and Fig. S5.2 [10] ]. These data support the hypothesis that protein dynamics is governed by the solvent motion [34], whereby water inside the protein "lubricates" the transitions between conformations without significant free-energy costs [25].

Similarly, at high $T$ [in Figs. 2(c) and 2(d)] ranging from low to high $\phi$, PGK also undergoes a single pressure-denaturation transition, but it is between the $\mathrm{C}$ and $\mathrm{U}$ states. Because of the increase in $T$, the $\mathrm{U}$ state is entropically more favorable than all other states. As such, the U state's entropy considerably compensates the C state's energy, causing an increase in the free-energy barrier between $\chi=0$ (C state) and 0.8-0.9 (U state) in Fig. 2(d).

From these $P-\phi$ slices at various $T$, our model predicts that crowding causes the folding of PGK a two-state transition, whereas the lack of crowding produces a multistate transition below a critical temperature $T_{c}$. Therefore, PGK undergoes a critical transition by either of two directions on the $T-P-\phi$ phase space. One direction is by increasing $\phi$ at low $T$ and sufficiently high $P$ surpassing a critical volume fraction $\phi_{c}$, as shown in Fig. 2(a) at the red critical point. This transition is seen clearly by the diminishing of the free-energy barrier in Fig. 2(b) pointed out by an orange arrow. The difference between the $\langle\chi\rangle$ 's of the $\mathrm{I}$ and $\mathrm{U}$ phases also disappears approaching $\phi_{c}$; i.e.,

$$
\lim _{\phi \rightarrow \phi_{c}^{-}}\left[\chi_{U}(\phi)-\chi_{I}(\phi)\right]=0,
$$

where $\phi_{c}$ is between 0.2 and 0.4 . The second way is by increasing $T$ at low $\phi$ and sufficiently high $P$ surpassing a critical temperature $T_{c}$. When $\phi=0$, the free-energy barrier observed in Fig. 2(b) by an orange arrow must diminish in order for the multistate free energy to become two state, resembling the high- $T$ free energy shown in Fig. 2(d). This result also means

$$
\lim _{T \rightarrow T_{c}^{-}}\left[\chi_{U}(T)-\chi_{I}(T)\right]=0 .
$$

Thus, these two directions that cause a critical transition indicate that $T_{c}$ is a line on $T-P-\phi$ phase space and the value decreases as $\phi$ increases.

\section{B. Experimental $T-P-\phi$ phase diagram of PGK}

To validate the computed phase diagram, we measure the $P-T$ phase diagram of PGK at various Ficoll 70 crowder concentrations to obtain the full $P-T-\phi$ information experimentally (Fig. 3). Whereas one cannot expect the exact temperatures and pressures to agree, identical topologies between the computational and experimental phase diagrams validate the general conclusions from simulations. As the mean fluorescence wavelength of tryptophan is sensitive to water exposure, changes in the states of PGK are detected by tryptophan fluorescence as the protein unfolds. The emission spectrum from tryptophan is strongly dependent on its (local) environment. Of the two states (excited and ground) that contribute to the emission, the excited state is highly perturbed by hydrogen bonding and the overall polarity of the solvent. The excited state of tryptophan has a dipole moment that differs from that of the ground state, and photoexcitation causes a rapid shift in the dipole moment, which then causes the surrounding water molecules to reorganize in order to stabilize the excited 
(a)

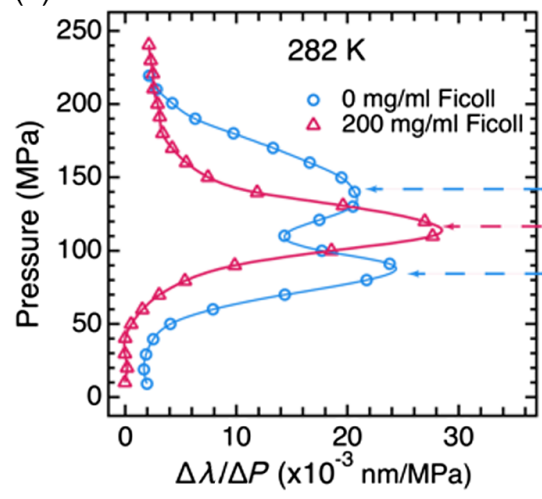

(b)

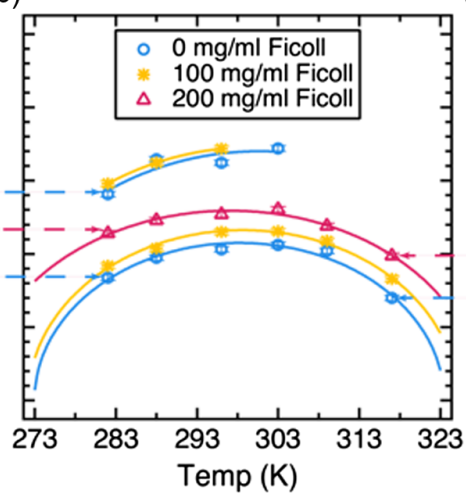

(c)

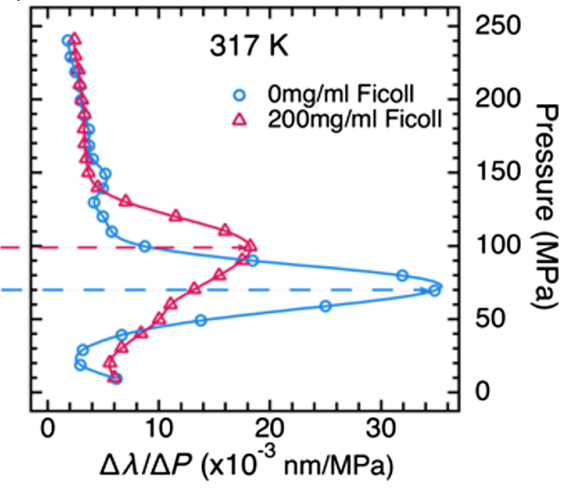

FIG. 3. Experimental $T-P-\phi$ phase diagram of PGK (full data in Ref. [10]). (a) The derivative of the mean tryptophan fluorescence wavelength vs the pressure of PGK at $282 \mathrm{~K}$ calculated from fluorescence spectra. Two of six Ficoll 70 concentrations are shown. The markers show the data points, and the solid line shows a cubic spline interpolation. The blue curve $(0 \mathrm{mg} / \mathrm{ml} \mathrm{Ficoll} 70)$ has two peaks as the pressure increases, signifying two transitions; the magenta curve $(200 \mathrm{mg} / \mathrm{ml}$ Ficoll 70$)$ has only one peak point, signifying only one transition when pressure is applied. The dashed lines point from transition midpoints to the corresponding point in the phase diagram. (b) $P$-T phase diagrams at several $\phi$ obtained by fitting the fluorescence data to obtain the inflection points of $\lambda_{m}(P)$ (peaks in the derivative $\partial \lambda_{m} / \partial P$ ). Three of six Ficoll 70 concentrations are shown. Circles represent midpoint pressures measured at 282, 288, 296, 303, 309, and $317 \mathrm{~K}$ in absence of Ficoll $70(0 \mathrm{mg} / \mathrm{ml})$, asterisks represent transitions for the middle Ficoll 70 concentration $(100 \mathrm{mg} / \mathrm{ml})$, and triangles represent transitions for the highest Ficoll 70 concentration $(200 \mathrm{mg} / \mathrm{ml})$. At high $T$, or upon increasing the Ficoll 70 concentration, the second (higher $P$ ) transition disappears, mapping out a critical point that moves to a lower $T_{c}$ at a higher Ficoll 70 concentration. Solid elliptical curves going through the circles are fits to Eq. (4) representing the $\Delta G=0$ curves. (c) Equivalent data as in (a) at $317 \mathrm{~K}$. Note that the second (higher $P$ ) transition is never present at high $T$.

tryptophan $[35,36]$. When the tryptophan is in a nonpolar environment, buried within the core of the protein (as it is when the protein is folded), there is less stabilization of the excited state. When the polar side chain of tryptophan is exposed to a polar solvent such as water, solvent rearrangement lowers the energy of the excited state, thus resulting in a redshift (Stokes shift) of the emission. Thus, the mean fluorescence wavelength increases upon unfolding of the protein due to the increase in exposure to water. Tryptophan fluorescence is also affected by a number of other factors including interactions with the charged side chains, though in our experience the polar solvent exposure is the major reason for the redshift upon unfolding [35,37]. We scan $T$ from 283 to $318 \mathrm{~K}$ at constant $P$, and $P$ from 0 to $250 \mathrm{MPa}$ at constant $T$, using $0,25,50$, 100,150 , and $200 \mathrm{mg} / \mathrm{ml}$ of Ficoll 70 concentrations ( $\phi=0$ to approximately 0.56 ) to cover the complete phase diagram. Each transition produces a sigmoidal step in the plot of mean tryptophan fluorescence wavelength $\lambda_{m}$ vs $P$ (Fig. S2.1 [10]).

In the absence of the crowder and at sufficiently low $T$ [Fig. 3(a), blue trace], there are two steps in $\lambda_{m}$ as a function of $P$, signifying two separate transitions among three states. These steps are straightforwardly revealed by plotting $\partial \lambda_{m} / \partial P$ and identifying peaks [see Figs. 3(a) and S2.1 [10]). We assign the first peak to the C-to-I transition and the second to the I-to-U transition. At sufficiently high $T$, at $\geq 303 \mathrm{~K}$ and $P \approx 170 \mathrm{MPa}$, one of the peaks disappears [Fig. 3(c), blue trace], leaving only one transition between two states. We assign it to a direct transition from $\mathrm{C}$ to $\mathrm{U}$, as shown in Fig. 2(c), corresponding to a critical point at $T_{c}=306 \pm 3 \mathrm{~K}$. When the crowder is added, $T_{c}$ moves to a lower temperature until the apparent three-state transition is no longer observed at $200 \mathrm{mg} / \mathrm{ml}$ Ficoll 70 [Figs. 3(a) and 3(c), red traces]. We assign it to the transition between $\mathrm{C}$ and SU/SPH/CC as shown in Fig. 2(a). Accurate transition midpoints $\left(T_{m}, P_{m}\right.$, and $\left.\phi_{m}\right)$ are obtained from each trace by fitting to sigmoidal two- or three-state models [solid curves in Figs. 3(a) and 3(c); see Appendix B; all data traces are shown in Ref. [10], Sec. S4). Singular value decomposition analysis (Ref. [10], Sec. S4) also strongly supports the conclusions obtained from analyzing $\lambda_{m}$.

We construct $P-T$ planes of the phase diagram at all crowder concentrations as follows: First, the transition midpoints are plotted on $P-T$ slices at constant $\phi$ as shown in Fig. 3(b). These points correspond to a zero free-energy difference $(\Delta G=0)$ for the first-order transition, where concentrations of $\mathrm{C}$ and I, I and $\mathrm{U}$, or $\mathrm{C}$ and $\mathrm{U}$ (depending on the location on the phase diagram) are equal. Then, the transitions are fitted to Hawley's elliptical $P-T$ phase curve for proteins [38]:

$$
\begin{aligned}
\Delta G(T, P)= & \frac{1}{2} \Delta \beta\left(P-P_{0}\right)^{2}+\Delta \alpha\left(T-T_{0}\right)\left(P-P_{0}\right) \\
& -\Delta C_{P}\left[T\left(\ln \frac{T}{T_{0}}-1\right)+T_{0}\right] \\
& +\Delta V_{0}\left(P-P_{0}\right)-\Delta S_{0}\left(T-T_{0}\right)+\Delta G_{0},
\end{aligned}
$$


at each value of $\phi$ (fits for all $\phi$ and parameter definitions in Ref. [10], Sec. S4). Here $\Delta \beta, \Delta \alpha, \Delta C_{P}, \Delta V_{0}$, and $\Delta S_{0}$ signify changes in the compressibility, thermal expansion coefficient, heat capacity, volume, and entropy, respectively. The resulting experimental phase diagram in Fig. 3(b) agrees with the computational data: (i) Both exhibit two pressure transitions at low $T$, (ii) one at high $T$, and (iii) a shift from two to three transitions at a value of $T_{c}$ that decreases with increased crowding.

The simulation predicts that, in the state $\mathrm{I}$, the $\mathrm{N}$ terminus would be unfolded and the $\mathrm{C}$ terminus folded. We truncate the protein to the $\mathrm{N}$-terminal domain and indeed find it to be unfolded with a long tryptophan fluorescence wavelength and no cooperative transition (Fig. S4.4 [10]). Moreover, the C-terminal domain of PGK is stable by itself [39]. Combined, these two observations strongly support the computational assignment of the I state with the N-terminal domain primarily unfolded and the C-terminal domain mostly folded. Therefore, our experimental data and simulations are in agreement as evidenced by the disappearance of the difference between two phases at high $T$ or high $\phi$ as well as the general structural features of the I state formed at low crowding.

\section{Unified $T-P-\phi$ phase diagram of PGK}

The three-dimensional (3D) $T-P-\phi$ phase diagram in Fig. 4(c) presents a unified picture of the computational and experimental results. This $3 \mathrm{D}$ phase diagram includes two surfaces: The blue surface represents the phase boundary bordering the $\mathrm{C}$ phase, and the red surface represents I-U coexistence surfaces (the calculations of the surfaces can be found in Appendix C and Ref. [10], Sec. S6). The projection of this 3D coexistence surface onto a 2D $\phi$ - $P$ plane in Fig. 4(a) shows a low- and high- $T$ slice similar to that found computationally in Fig. 2. When projected on the $P-T$ plane in Fig. 4(b), it shows a low- $\phi$ and high- $\phi$ slice as found experimentally in Fig. 3. As the temperature increases, the second transition surface terminates at a critical line [bold red line on the red I-U coexistence surface in Fig. 4(c)]. As the crowding volume fraction increases, the critical point on each $P-T$ slice shifts toward lower temperatures. Thus, from the experiment, above $\phi=\phi_{c} \approx 0.5$ or $T=T_{c}^{0} \approx 306 \mathrm{~K}$, the pressure-induced folding transition contains only two apparent phases, whereas at low $\phi$ and $T$, PGK exhibits apparent three-state folding.

To quantitate the unified 3D phase diagram, we modify Hawley's theory [Eq. (4)] by incorporating the free-energy change due to crowding $\Delta G_{\text {crowd }}(\phi)$, using a first-order approximation to Minton's theory [16], to construct the first transition surface [blue surface in Fig. 4(c)]. Just as the curves in Fig. 3 correspond to a zero free-energy change, this surface represents $\Delta G(T, P, \phi)=\Delta G(T, P)+$ $\Delta G_{\text {crowd }}(\phi)=0$, where

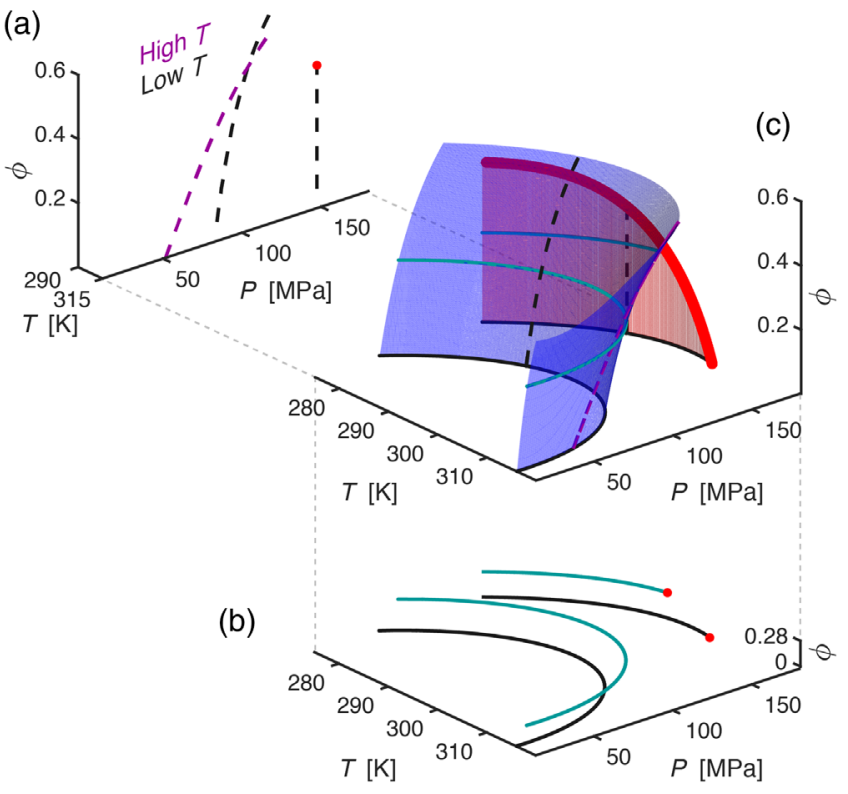

FIG. 4. $\quad T-P-\phi$ phase diagram of PGK from the theory mapped onto the experimental data. (a) PGK's $\phi-P$ phase plane at high (magenta) and low (black) $T$. Dotted lines represent the division between distinct configurational phases. The red dot signifies a critical point. (b) Slices of the $P-T$ phase diagram observed experimentally at no Ficoll 70 (black curve) and $100 \mathrm{mg} / \mathrm{ml}$ Ficoll 70 (cyan curve). Note that I-U coexistence curve terminates at the critical point (in red dots) and shifts $T_{c}$ to a lower temperature in the presence of Ficoll 70. Solid elliptical curves going through the circles are the fits representing the $\Delta G=0$ curves. (c) A $T-P-\phi$ phase diagram of PGK. The blue surface is the coexistence surface bordering the $\mathrm{C}$ phase (C-I, C-U, or $\mathrm{C}-\mathrm{SPH} / \mathrm{CC} / \mathrm{SPH}$ depending on $T$ and $\phi$ ), and the red surface is the I-U coexistence surface. The dashed magenta and black lines are the $\phi-P$ cross section from Fig. 4(a), and the solid black and cyan lines are the $P-T$ cross section from Fig. 4(b). The bold, red line bordering the red surface is the critical line.

$$
\Delta G_{\text {crowd }}(\phi)=g\left(\frac{\phi}{1-\phi}\right)+\mathcal{O}\left(\phi^{2}\right) .
$$

The constant $g$ takes into account the covolume change of the protein, demonstrating that the covolume and crowding are thermodynamic conjugates.

As for the critical line on the second transition surface [in red in Fig. 4(c)], we use scaling arguments to derive the equation for the critical line:

$P-P_{c}=a_{1}\left[T-T_{c}(\phi)\right]+a_{2}\left[T-T_{c}(\phi)\right]^{2}+\mathcal{O}\left(\Delta T^{3}\right)$,

by treating the protein's U-to-I transition similar to in the coil-globule transition of the theory $[40,41]$. Here,

$$
T_{c}(\phi)=T_{c}^{0}\left(1-\frac{\phi}{\phi_{c}}\right)^{\gamma},
$$

where $T_{c}^{0}$ is the critical temperature without crowding, $\phi_{c}$ is the critical crowding volume fraction, $P_{c}$ 
(approximately equal to $170 \mathrm{MPa}$ ) is the critical pressure taken from our experiment at $T_{c}^{0}$, and $a_{1}=\left.(d P / d T)\right|_{T=T_{c}^{0}}$ and $a_{2}=\left.\left(d^{2} P / d T^{2}\right)\right|_{T=T_{c}^{0}}$. From the fitting to experimental critical points at all slices of $\phi$, we find $\gamma=$ $0.40 \pm 0.01$, which is the predicted scaling exponent of a polymer collapse due to crowders, $\gamma=2 / 5[42,43]$ (see Appendix C and Ref. [10], Sec. S6). From this phase diagram, we can see that the protein moves through a diverse phase space, suggesting different folding mechanisms that depend on how the phase diagram is traced out $[44,45]$.

\section{Consequences of criticality}

In Fig. 5, we explore the impact of $P$ and $\phi$ on the folding of PGK. The consequences of the critical regime are revealed by the ensemble distributions of the cavity volume (conjugate variable of $P$ ) and covolume (conjugate variable of osmotic pressure, which is related to $\phi$ ) $[15,16]$ from our simulations (also see Fig. S5.1 [10]). In the critical regime, small perturbations in crowding $\phi, P$, or $T$ significantly affect the system.

We investigate the response of the conformational distribution of structures close to the critical region by comparing the cavity volume fluctuations $\left(\delta V^{2}=\right.$ $\left\langle V^{2}\right\rangle-\langle V\rangle^{2}$ ) (or, proportionally, the compressibility) and structural fluctuations $\left(\delta \chi^{2}=\left\langle\chi^{2}\right\rangle-\langle\chi\rangle^{2}\right)$ in the presence and absence of a crowding agent. PGK has larger $\delta V$ [Fig. 5(a)] at $\phi=0.4$ with a peak at $6.6 \epsilon_{0} / \sigma^{3}$ $\left(\epsilon_{0} / \sigma^{3} \approx 76 \mathrm{MPa}\right)$ than that of $\phi=0$. We suspect that the critical regime is between $\phi=0.2$ and 0.4 and between pressures $4.6 \epsilon_{0} / \sigma^{3}$ and $6.6 \epsilon_{0} / \sigma^{3}$ at a temperature of $0.97 \epsilon_{0} / k_{B} T$ in the computational model, which qualitatively agrees with the experiment. Even though $\delta \chi$ is large in the presence of crowders, structures lie in a narrow range of covolumes, making them indistinguishable to macromolecular crowding effects if the shape can be neglected to the zeroth order [Fig. 5(b)]. A sample of the diverse structures with similar cavity volumes and covolumes is shown in Fig. 5(c).

Not only does crowding shift the population of structures to more compact states such as CC or SPH (Fig. 2 and Ref. [12]), where the two ligand-binding sites (for ADP and 1,3-DPG) come into close proximity of each other, but it also increases the structural fluctuations of the compact states by bringing PGK closer to the critical regime, as shown in Fig. 5. Both of these properties would be likely to facilitate enzymatic activity. This conjecture is corroborated by previous FRET experiments that show an increase in PGK's enzymatic activity as the Ficoll 70 concentration increases at $293 \mathrm{~K}$ and ambient $P$ [12]. Together, these data suggest that criticality contributes to the enzymatic function of a protein.

\section{CONCLUSION}

In summary, we have shown direct evidence of equilibrium critical-like behavior on the $T-P-\phi$ phase diagram of a

(b)

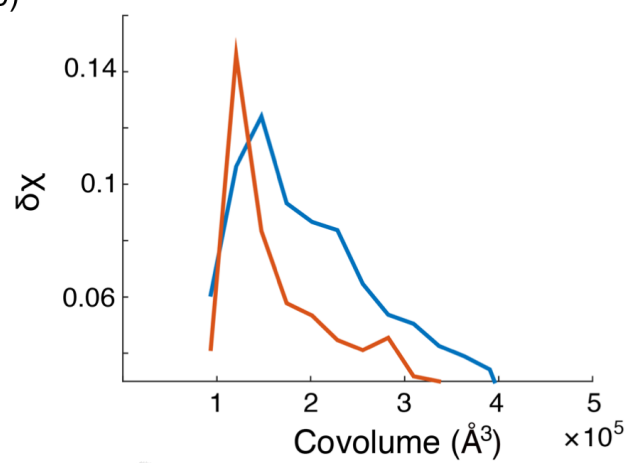

(c)
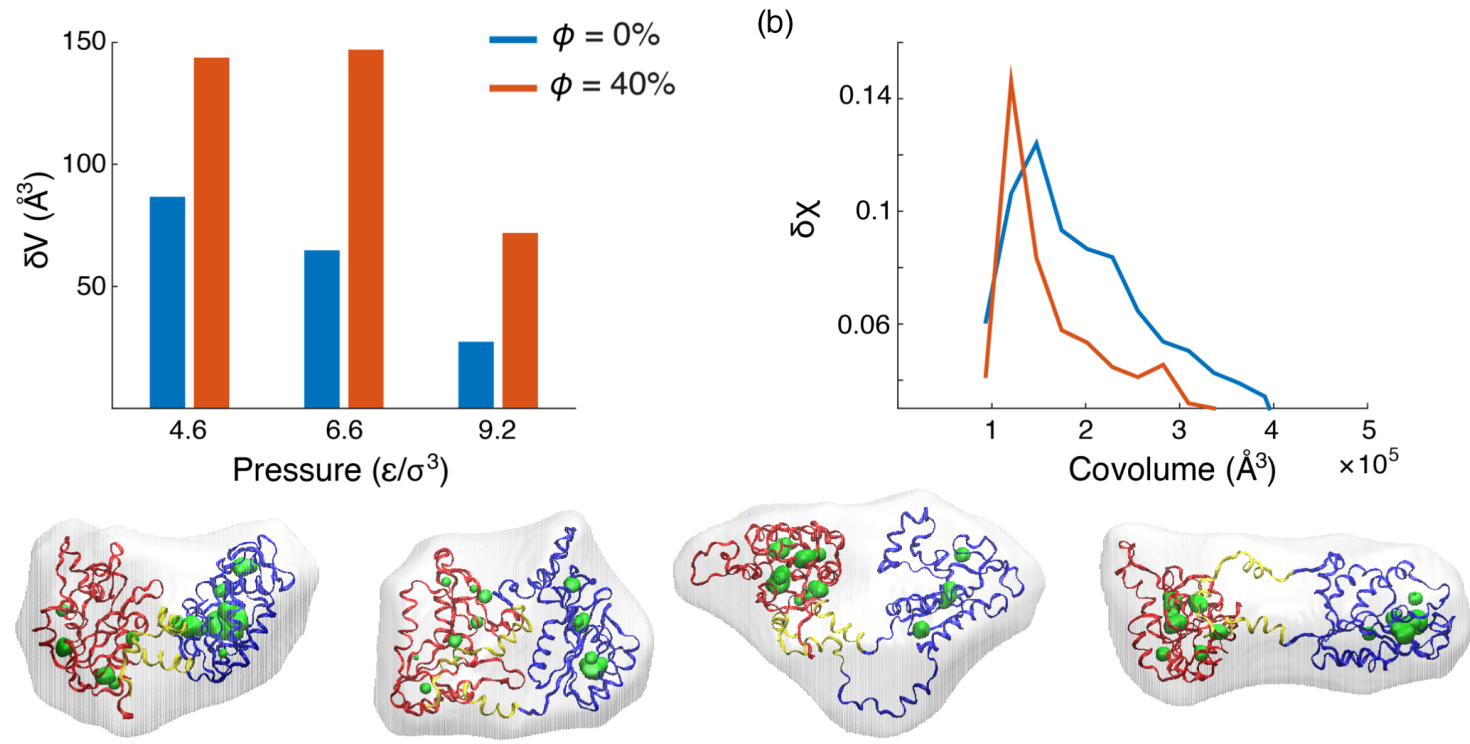

FIG. 5. Cavity volume and structural fluctuations near the critical regime. (a) Cavity volume fluctuations $\delta V^{2}=\left\langle V^{2}\right\rangle-\langle V\rangle^{2}$ (or proportionally compressibility) of PGK at $k_{B} T / \epsilon_{0}=0.97$ and $\sigma^{3} P / \epsilon_{0}=4.6,6.6$, and 9.2 with (orange line) and without (blue line) crowding $\left(\epsilon_{0} / \sigma^{3} \approx 76 \mathrm{MPa}\right)$. (b) Overlap fluctuations $\delta \chi^{2}=\left\langle\chi^{2}\right\rangle-\langle\chi\rangle^{2}$ as a function of the covolume at pressure $\sigma^{3} P / \epsilon_{0}=6.6$. (c) Conformations from the ensemble in the presence of crowding at $k_{B} T / \epsilon_{0}=0.97$ and $\sigma^{3} P / \epsilon_{0}=6.6$ with covolumes approximately equal to $1.1 \times 10^{5} \AA^{3}$ and cavities approximately equal to $200 \AA^{3}$. The leftmost conformation is a crystal state. The following structures from left to right have $\mathrm{a} \chi=0.31,0.38$, and 0.48 . The $\mathrm{N}$ and $\mathrm{C}$ domains and hinge are in red, blue, and yellow, respectively. Covolumes are shown as translucent surfaces surrounding the protein, and cavity surfaces are shown in green. 
protein by computational simulations, by fluorescence spectroscopy, and by a theoretical argument based on polymer physics. Despite the simplicity of the computational and theoretical model, all three different approaches agree with one another, validating the trends on the $T-P-\phi$ phase diagram and the presence of the critical regime. Above the critical line in Fig. 4(c) (by increasing $T, \phi$, or both at $P_{c} \approx 170 \mathrm{MPa}$ ), the difference between the $\mathrm{I}$ and $\mathrm{U}$ phases disappears. This effect is due to the loss of the freeenergy barrier between the two phases [orange arrow in Fig. 2(b)] and is reaffirmed by the high-pressure fluorescence measurements (Fig. 3).

What is the origin of the critical behavior in proteins? The answer to this question relies on weaving two concepts together. First, proteins are biopolymers that often undergo an abrupt or first-order-like transition to a compacted folded state from an expanded unfolded state or coil at a folding temperature $T_{F}$. Second, the coil-globule transition seen in other polymers is a continuous transition at a specific temperature called the $\theta$ temperature, $T_{\theta}[40,41]$. Therefore, the first-order transition in protein folding must be occurring near the collapse transition $\left(T_{F} \approx T_{\theta}\right)$, meaning it normally is tricritical [7]. In the current system, the pressure perturbation may cause $T_{F} \neq T_{\theta}$, separating the continuous and first-order transitions. When going from a continuous to a first-order transition, there are signatures of passing through a critical point $[46,47]$. Moreover, when $\phi$ is high $\left(\phi>\phi_{c}\right)$, the protein is already collapsed even when it is unfolded. Our theoretical model in Eq. (7) and Appendix C (also Ref. [10], Sec. S6) captures this postulation of the basis of criticality in proteins.

Furthermore, our computational and experimental results are in accord with the capillarity picture of folding [48], which posits a wetting interface between folded and unfolded parts of a protein, giving rise to a diverse phase space. Strong macromolecular crowding, which drives conformational changes to favor compact states, roughens that wetting interface, allowing cavities to spread throughout the conformation of the protein, with two major consequences. A roughened interface reduces activation barriers for folding, driving multistate transitions toward apparent two-state transitions. It also creates a critical state where heterogeneous conformations coexist, as the front of wetting interface moves across the protein.

We conclude that large structural fluctuations (Fig. 5) and the merging of protein phases are consequences of being close to a critical point [Fig. 4(c)]. At such a point, the barrier separating states vanishes (here, between I and U). Critical behavior has been proposed for protein folding at the onset of downhill folding [8], but its manifestation has been challenging to demonstrate computationally and experimentally [49]. Macromolecular crowding shifts the critical point to a lower temperature [Eq. (7)], indicating that such criticality could be physiologically important [3,4]. While the critical point at $305 \mathrm{~K}$ and
$170 \mathrm{MPa}$ lies in a region of the phase diagram most relevant to piezophiles (high-pressure-thriving organisms), the effects of criticality extend to lower pressures for several reasons. First, since critical fluctuations remain fairly large and decay with a heavy tail (or power law) when moving away from the critical point [50], the mere existence of the critical point has far-reaching consequences across a wide range of $T, P$, and $\phi$. Furthermore, this effect is even more pronounced for nanoscale objects like individual proteins, because their phase transitions are broadened by finite-size effects. For example, a $50 \mathrm{MPa}$ pressure change and a $10 \mathrm{~K}$ temperature change are roughly equivalent to a tiny approximately $10 \mathrm{~kJ} / \mathrm{mol}$ free-energy change [51]. The volume of unfolding and entropy of unfolding for proteins are quite small. Thus, critical points may indirectly affect the boundaries and structural fluctuations of nearby phases, which could be advantageous for processes (e.g., enzyme catalysis) dependent on accessing a wide range of conformations with smaller activation barriers for functional purposes inside the cell. Note, though, that the majority of organisms on Earth by population size ( $15 \%$ by biomass) are extremophilic bacteria and archaea living at high pressure and/or boiling conditions $[30,52]$.

Further work is needed to provide stronger evidence for the universality of critical behavior in proteins. Because of their complexity, proteins are not like other conventional condensed matter systems, and conventional tools, such as finite-size scaling [53] or renormalization group theory [54], are not clearly applicable, although recent efforts are making strides with these methods to tame similarly complex systems [55,56]. One may extend our analysis to other proteins using an example of universality as inspiration, such as Guggenheim's plot of the law of corresponding states [57]. In principle, proteins may obey such a law. By measuring the critical points on $T-P-\phi$ space (using a crowder that is a semirigid or hard sphere, such as Ficoll 70) for different proteins and scaling $T$ and an order parameter (such as density) correctly, the binodal lines should collapse into a single, universal curve. However, there are several complications to consider: (i) The scaling of $T$ and the order parameter may not be as simple due to finite-size corrections [58], (ii) $T_{c}$ or $\phi_{c}$ may equal 0 (say, for intrinsically disordered proteins [59]) or be infinite (say, for rigid scaffold proteins), (iii) the barrier between the two phases that disappears under the critical transition may not necessarily be between the I and U phases, and (iv) the universality classes of proteins may depend on their functions. The current investigation is a starting point toward developing universal principles of protein folding relevant to the environmental perturbations inside living cells and is an inspiration to create new tools to understand critical phenomena in these complex systems. 


\section{ACKNOWLEDGMENTS}

We thank Professor Dave Thirumalai, Professor Vassiliy Lubchenko, Professor Greg Morrison, Professor Peter G. Wolynes, Dr. Anna Jean Wirth, and Dr. Gregory P. Gasic for helpful and stimulating discussions. M. B.P. was supported by a Howard Hughes Medical Institute International Student Research Fellowship and then a Helen Hay Whitney Foundation Postdoctoral Fellowship. A. G. G is supported by a training fellowship on the Houston Area Molecular Biophysics Program (T32 GM008280). M. S. C. and A. G. G. were funded by the National Science Foundation (MCB-1412532, PHY1427654 , and OAC-1531814) and thank computing resources from the Center for Advanced Computing and Data Systems at UH. M. G. and M. B. P. were funded by National Institute of Health Grant No. GM093318.

\section{APPENDIX A: SIMULATION MODEL}

Our simulations use a structure-based model, which is a minimalist protein model ("beads on a chain") that incorporates experimentally derived structural information [60], to investigate the mechanism of protein folding dynamics optimally. The emergence of structure and function from a protein sequence makes the modeling of proteins from first principles (ab initio models) computationally and theoretically prohibitive. Therefore, experimentally derived structural information is needed (even in models termed "all atom," which refine $a b$ initio force field parameters to fit experimentally known structures) to capture key features in protein folding and dynamics [61]. A structure-based model is often utilized as the "ideal gas" of protein folding for the investigation of a wide range of folding mechanisms $[2,62]$. This model renders an energy landscape [63] with minimal frustration and contains a dominant basin of attraction, corresponding to an experimentally determined configuration [64]. As such, the model carries the bonus of being computationally inexpensive, enabling long-timescale simulations to be obtained for a large protein and macromolecular crowding system. Long-timescale simulations are also crucial for high-pressure unfolding, since pressure unfolds proteins at an order of magnitude (or more) slower than heat unfolding; therefore, structurebased, minimalist-model simulations provide statistically significant results. Lastly, structure-based models tend to capture unfolded protein scaling laws better than all-atom models [65], which are necessary to characterize the various noncrystal states of PGK correctly.

Similar to adding specific complexity to the ideal gas model to study specific phenomena, we add the desolvation barrier [25] to the native interactions that account for the free-energy cost to expel a water molecule in the first hydration shell between two hydrophobic residues [66]. This approach allows us to study pressure unfolding, leading to the appearance of a partially folded intermediate. The use of this model has been validated in other systems [28]. The total system is described by the Hamiltonian $\mathcal{H}_{\text {tot }} \equiv \mathcal{H}_{p}+\mathcal{H}_{p c}+\mathcal{H}_{c}$, which accounts for the interaction within the protein $\left(\mathcal{H}_{p}\right)$, between the protein and crowders $\left(\mathcal{H}_{p c}\right)$, and between crowders $\left(\mathcal{H}_{c}\right)$. The Hamiltonian of this structure-based protein model, $\mathcal{H}_{p}$, is as follows:

$$
\begin{aligned}
\mathcal{H}_{p}\left(\Gamma, \Gamma^{0}\right)= & \sum_{i<j} K_{r}\left(r_{i j}-r_{i j}^{0}\right)^{2} \delta_{j, i+1}+\sum_{i \in \text { angles }} K_{\theta}\left(\theta_{i}-\theta_{i}^{0}\right)^{2}+\sum_{i \in \text { dihedrals }} K_{\phi}\left(\left\{1-\cos \left[\phi_{i}-\phi_{i}^{0}\right]\right\}+\frac{1}{2}\left\{1-\cos \left[3\left(\phi_{i}-\phi_{i}^{0}\right)\right]\right\}\right) \\
& +\sum_{i, j \in \text { native }} U\left(r_{i j}, \epsilon, \epsilon^{\prime \prime}\right)+\sum_{i, j \notin \text { native }} \epsilon_{0}\left(\frac{\sigma}{r_{i j}}\right)^{12},
\end{aligned}
$$

where $\Gamma$ is a configuration of the set $r, \theta, \phi$. The $r_{i j}$ term is the distance between $i$ th and $j$ th residues, $\theta$ is the angle between three consecutive beads, and $\phi$ is the dihedral angle defined over four sequential residues. $\delta$ is the Kronecker delta function. $\Gamma^{0}=$ $\left\{\left\{r^{0}\right\},\left\{\theta^{0}\right\},\left\{\phi^{0}\right\}\right\}$ is obtained from the crystal structure configuration. Lastly, $U\left(r_{i j}, \epsilon, \epsilon^{\prime \prime}\right)$ is the desolvation potential in Fig. 1(b) (or Fig. S2.2 [10]), which contains a $P$-dependent contact well energy $(\epsilon)$ and barrier height energy $\left(\epsilon^{\prime \prime}\right)$ as

$$
\begin{gathered}
\epsilon(P)=\epsilon_{0}-\xi_{1} P, \\
\epsilon^{\prime \prime}(P)=\epsilon_{0}^{\prime \prime}+\xi_{2} P,
\end{gathered}
$$

where $\epsilon_{0}$ is the solvent-averaged energy and $\epsilon_{0}^{\prime \prime}$ is the barrier height at ambient $P$. The constants $\xi_{1}$ and $\xi_{2}$ are taken from Ref. [66].

Crowders are modeled as hard spheres with Hamiltonians $\mathcal{H}_{p c}$ and $\mathcal{H}_{c}$ with the following form:

$$
\begin{gathered}
\mathcal{H}_{p c}\left(r_{i j}\right)=\sum_{i}^{N} \sum_{j}^{n_{c}} \epsilon_{0}\left(\frac{\sigma_{i j}}{r_{i j}}\right), \\
\mathcal{H}_{c}\left(r_{i j}\right)=\sum_{i<j}^{n_{c}} \epsilon_{0}\left(\frac{\sigma_{i j}}{r_{i j}}\right),
\end{gathered}
$$

where $N$ and $n_{c}$ are the number of residues $(=415)$ and crowders, respectively; $\sigma_{i j}=0.5\left(\sigma_{i}+\sigma_{j}\right)$ is the distance between any two particles in direct contact. 
The complete descriptions of a structure-based protein model, desolvation potential, and simulations of PGK in a periodic cubic box of Ficoll 70 are provided in Supplemental Material [10]. All simulations are performed using GROMACS2016.3 molecular dynamics software [67].

\section{APPENDIX B: HIGH-PRESSURE FLUORESCENCE EXPERIMENT METHODS}

Fluorescence experiments are carried out at high pressure using a high-pressure cell (ISS High-Pressure Cell System) on a fluorimeter (JASCO FP8300). A computerized high-pressure generator (Pressure BioSciences Inc. HUB440) is used to pressurize the fluorescence cell. We use a rectangular quartz cuvette with a path length of $6 \mathrm{~mm}$, and deionized water is used as the pressurizing fluid. The pressure is raised from 0.1 to $250 \mathrm{MPa}$ at a rate of $10 \mathrm{MPa} / \mathrm{min}$ and held at intervals of $10 \mathrm{MPa}$ for a $5 \mathrm{~min}$ wait time to allow sample equilibration. Fluorescence spectra from 300 to $450 \mathrm{~nm}$ are acquired in the middle of the wait time, and an in-built proportional-integralderivative feedback loop is used to obtain accurate pressures (within 5 bars of target pressure). The temperature is controlled using an external water-circulating bath. To construct a complete $T-P-\phi$ phase diagram, fluorescence measurements are done at six different Ficoll 70 concentrations $([$ Ficoll 70$]=0,25,50,100,150$, and $200 \mathrm{mg} / \mathrm{ml}$ ), each at six different temperatures $T$ ranging from 282 to $317 \mathrm{~K}\left(9^{\circ} \mathrm{C}\right.$ to $\left.44^{\circ} \mathrm{C}\right)$. Equilibrium traces of mean fluorescence wavelength vs pressure [e.g., Fig. 3(a)] are fit to a two-state or three-state thermodynamic model (see Supplemental Material [10]), depending on whether the derivative (Fig. S1.2 [10]) of the titration curve identifies one or two transitions (an inflection point in the fluorescence vs $P$ trace at given $T$ and [Ficoll 70] produces a peak in the derivative). The fitted transition midpoints $\left(P_{m}, T_{m}, \phi_{m}\right)$ are then plotted in a phase diagram [e.g., Fig. 3(b)] and fitted to Eq. (4). See Ref. [10] for complete data and fitting parameters.

\section{APPENDIX C: CONSTRUCTION OF THE PHASE DIAGRAM}

We derive the critical line [Eqs. (6) and (7); red line in Fig. 4(c)] on the $T-P-\phi$ phase diagram using arguments based on the coil-globule transition [40,41] of a polymer. Beginning with a Landau-Ginsberg free energy [68], $F=-r(T, \phi) \Psi^{2}+u \Psi^{4}+F_{0}$ to describe the critical transition, where $\Psi$ is the order parameter, which is a scaled and shifted $R_{g}$ (radius of gyration) so that $\Psi=-\Psi_{0}$ for the I state and $\Psi=+\Psi_{0}$ for the U state. Since pressure is involved only with the first-order transitions, it can be ignored for now. At the critical temperature, the barrier between the I and U states vanishes, meaning $r=0$; therefore, a reasonable function is $r(T, \phi)=-r_{0}\left[T-T_{c}(\phi)\right]$, where the critical temperature
$T_{c}$ is a function of $\phi$ and $r_{0}$ is a positive constant. To find the $\phi$ dependence of $T_{c}$, we used the scaling relationship

$$
\frac{R_{g}(\phi)^{2}}{R_{g}(0)^{2}} \sim\left(1-c_{0} \phi\right)^{\gamma}
$$

which relates $R_{g}$ at a given $\phi$ to $R_{g}$ without crowders for the collapse of a coil-to-globule transition [42]. The scaling exponent $\gamma$ is shown to be $2 / 5$ in Refs. $[42,43]$. Since the collapse of the polymer, or in the current case the protein, is dependent on $\phi$, and since $\Psi^{2} \sim$ $R_{g}(\phi)^{2} / R_{g}(0)^{2}$, the critical temperature $T_{c}(\phi)$ causing the free-energy barrier between I and $\mathrm{U}$ to disappear must also scale as Eq. (C1), giving Eq. (7) (see Ref. [10], Sec. S6, for more details). We fit Eq. (7) to the experimental critical point values at all Ficoll 70 concentrations to find $\gamma$ and $\phi_{c}$ (or $1 / c_{0}$ ). We fit Eq. (6) to experimental values of the I-to- $\mathrm{U}$ transition surface to find the Taylor expansion coefficients.

Lastly, we modify Hawley's equation [38] to fit the C-to-I (or $\mathrm{U}$, depending on $T$ and $\phi$ ) transition surface [in blue in Fig. 4(c)] by adding a $\phi$-dependent $\Delta G_{\text {crowd }}(\phi)$ term to Eq. (4), making the 3D free-energy change $\Delta G(T, P, \phi)=\Delta G(T, P)+\Delta G_{\text {crowd }}(\phi)$. This term is similar to Minton's theory [16], which treats the folded and unfolded proteins as effective hard spheres and employs the scaled particle theory to estimate the change in folding free energy as the difference between the insertion free energy for the folded and the unfolded states. Equation (5) adds one more fitting parameter, $g$, to the total free-energy change compared to Eq. (4).

[1] J. P. Crutchfield, Between Order and Chaos, Nat. Phys. 8, 17 (2012).

[2] P. C. Whitford, K. Y. Sanbonmatsu, and J. N. Onuchic, Biomolecular Dynamics: Order-Disorder Transitions and Energy Landscapes, Rep. Prog. Phys. 75, 076601 (2012).

[3] T. Mora and W. Bialek, Are Biological Systems Poised at Criticality?, J. Stat. Phys. 144, 268 (2011).

[4] M. A. Munoz, Colloquium: Criticality and Dynamical Scaling in Living Systems, Rev. Mod. Phys. 90, 031001 (2018).

[5] P. G. Wolynes, J. N. Onuchic, and D. Thirumalai, Navigating the Folding Routes, Science 267, 1619 (1995).

[6] J. N. Onuchic, P. G. Wolynes, Z. Luthey-Schulten, and N. D. Socci, Toward an Outline of the Topography of a Realistic Protein-Folding Funnel, Proc. Natl. Acad. Sci. U.S.A. 92, 3626 (1995).

[7] M. S. Li, D. K. Klimov, and D. Thirumalai, Finite Size Effects on Thermal Denaturation of Globular Proteins, Phys. Rev. Lett. 93, 268107 (2004).

[8] S. S. Cho, P. Weinkam, and P. G. Wolynes, Origins of Barriers and Barrierless Folding in BBL, Proc. Natl. Acad. Sci. U.S.A. 105, 118 (2008). 
[9] Q.-Y. Tang, Y.-Y. Zhang, J. Wang, W. Wang, and D. R. Chialvo, Critical Fluctuations in the Native State of Proteins, Phys. Rev. Lett. 118, 088102 (2017).

[10] See Supplemental Material at http://link.aps.org/ supplemental/10.1103/PhysRevX.9.041035 for simulation and experiment details and further analysis.

[11] O. Miyashita, J. N. Onuchic, and P. G. Wolynes, Nonlinear Elasticity, Protein Quakes, and the Energy Landscapes of Functional Transitions in Proteins, Proc. Natl. Acad. Sci. U.S.A. 100, 12570 (2003).

[12] A. Dhar, A. Samiotakis, S. Ebbinghaus, L. Nienhaus, D. Homouz, M. Gruebele, and M.S. Cheung, Structure, Function, and Folding of Phosphoglycerate Kinase Are Strongly Perturbed by Macromolecular Crowding, Proc. Natl. Acad. Sci. U.S.A. 107, 17586 (2010).

[13] S. B. Zimmerman and S. O. Trach, Estimation of Macromolecule Concentrations and Excluded Volume Effects for the Cytoplasm of Escherichia Coli, J. Mol. Biol. 222, 599 (1991).

[14] S. Asakura and F. Oosawa, On Interaction between Two Bodies Immersed in a Solution of Macromolecules, J. Chem. Phys. 22, 1255 (1954).

[15] A.P. Minton, Excluded Volume as a Determinant of Macromolecular Structure and Reactivity, Biopolym. Orig. Res. Biomol. 20, 2093 (1981).

[16] A. P. Minton, Models for Excluded Volume Interaction between an Unfolded Protein and Rigid Macromolecular Cosolutes: Macromolecular Crowding and Protein Stability Revisited, Biophys. J. 88, 971 (2005).

[17] M. Gruebele, K. Dave, and S. Sukenik, Globular Protein Folding in vitro and in vivo, Annu. Rev. Biophys. 45, 233 (2016).

[18] T. Q. Luong, S. Kapoor, and R. Winter, Pressure: A Gateway to Fundamental Insights into Protein Solvation, Dynamics, and Function, ChemPhysChem 16, 3555 (2015).

[19] C. L. Dias, T. Ala-Nissila, M. Karttunen, I. Vattulainen, and M. Grant, Microscopic Mechanism for Cold Denaturation, Phys. Rev. Lett. 100, 118101 (2008).

[20] B. J. Sirovetz, N. P. Schafer, and P. G. Wolynes, Water Mediated Interactions and the Protein Folding Phase Diagram in the Temperature-Pressure Plane, J. Phys. Chem. B 119, 11416 (2015).

[21] T. G. Dewey, Fractals in Molecular Biophysics (Oxford University, New York, 1998).

[22] S. Reuveni, R. Granek, and J. Klafter, Proteins: Coexistence of Stability and Flexibility, Phys. Rev. Lett. 100, 208101 (2008).

[23] P. D. Chowdary and M. Gruebele, Molecules: What Kind of a Bag of Atoms?, J. Phys. Chem. A 113, 13139 (2009).

[24] J. Roche, J. A. Caro, D. R. Norberto, P. Barthe, C. Roumestand, J. L. Schlessman, A. E. Garcia, and C. A. Royer, Cavities Determine the Pressure Unfolding of Proteins, Proc. Natl. Acad. Sci. U.S.A. 109, 6945 (2012).

[25] M. S. Cheung, A. E. García, and J. N. Onuchic, Protein Folding Mediated by Solvation: Water Expulsion and Formation of the Hydrophobic Core Occur after the Structural Collapse, Proc. Natl. Acad. Sci. U.S.A. 99, 685 (2002).

[26] C. L. Dias and H. S. Chan, Pressure-Dependent Properties of Elementary Hydrophobic Interactions: Ramifications for
Activation Properties of Protein Folding, J. Phys. Chem. B 118, 7488 (2014).

[27] J.-E. Shea, J. N. Onuchic, and C. L. Brooks, Probing the Folding Free Energy Landscape of the src-SH3 Protein Domain, Proc. Natl. Acad. Sci. U.S.A. 99, 16064 (2002).

[28] A. Fernandez-Escamilla, M. Cheung, M. Vega, M. Wilmanns, J. Onuchic, and L. Serrano, Solvation in Protein Folding Analysis: Combination of Theoretical and Experimental Approaches, Proc. Natl. Acad. Sci. U.S.A. 101, 2834 (2004).

[29] D. Homouz, M. Perham, A. Samiotakis, M. S. Cheung, and P. Wittung-Stafshede, Crowded, Cell-like Environment Induces Shape Changes in Aspherical Protein, Proc. Natl. Acad. Sci. U.S.A. 105, 11754 (2008).

[30] F. A. Rainey and A. Oren, Extremophile Microorganisms and the Methods to Handle Them, in Methods in Microbiology (Elsevier, New York, 2006), Vol. 35, pp. 1-25.

[31] K. Luby-Phelps, P. E. Castle, D. L. Taylor, and F. Lanni, Hindered Diffusion of Inert Tracer Particles in the Cytoplasm of Mouse 373 Cells, Proc. Natl. Acad. Sci. U.S.A. 84, 4910 (1987).

[32] D. Venturoli and B. Rippe, Ficoll and Dextran vs. Globular Proteins as Probes for Testing Glomerular Permselectivity: Effects of Molecular Size, Shape, Charge, and Deformability, Am. J. Physiol. 288, F605 (2005).

[33] M. R. Shaw and D. Thirumalai, Free Polymer in a Colloidal Solution, Phys. Rev. A 44, R4797 (1991).

[34] P. W. Fenimore, H. Frauenfelder, B. H. McMahon, and F. G. Parak, Slaving: Solvent Fluctuations Dominate Protein Dynamics and Functions, Proc. Natl. Acad. Sci. U.S.A. 99, 16047 (2002).

[35] J. R. Lakowicz, Principles of Fluorescence Spectroscopy (Springer Science, New York, 2013).

[36] A. Ghisaidoobe and S. Chung, Intrinsic Tryptophan Fluorescence in the Detection and Analysis of Proteins: A Focus on Förster Resonance Energy Transfer Techniques, Int. J. Mol. Sci. 15, 22518 (2014).

[37] J. T. Vivian and P. R. Callis, Mechanisms of Tryptophan Fluorescence Shifts in Proteins, Biophys. J. 80, 2093 (2001).

[38] S. A. Hawley, Reversible Pressure-Temperature Denaturation of Chymotrypsinogen, Biochemistry 10, 2436 (1971).

[39] S. Osváth, J. J. Sabelko, and M. Gruebele, Tuning the Heterogeneous Early Folding Dynamics of Phosphoglycerate Kinase, J. Mol. Biol. 333, 187 (2003).

[40] I. Lifshitz, A. Y. Grosberg, and A. Khokhlov, Some Problems of the Statistical Physics of Polymer Chains with Volume Interaction, Rev. Mod. Phys. 50, 683 (1978).

[41] P.-G. De Gennes and P.-G. Gennes, Scaling Concepts in Polymer Physics (Cornell University, Ithaca, 1979).

[42] D. Thirumalai, Isolated Polymer Molecule in a Random Environment, Phys. Rev. A 37, 269 (1988).

[43] H. Kang, P. A. Pincus, C. Hyeon, and D. Thirumalai, Effects of Macromolecular Crowding on the Collapse of Biopolymers, Phys. Rev. Lett. 114, 068303 (2015).

[44] P. I. Zhuravlev, M. Hinczewski, S. Chakrabarti, S. Marqusee, and D. Thirumalai, Force-Dependent Switch in Protein Unfolding Pathways and Transition-State Movements, Proc. Natl. Acad. Sci. U.S.A. 113, E715 (2016). 
[45] C. A. Pierse and O. K. Dudko, Distinguishing Signatures of Multipathway Conformational Transitions, Phys. Rev. Lett. 118, 088101 (2017).

[46] M. P. Taylor, W. Paul, and K. Binder, All-or-None Proteinlike Folding Transition of a Flexible Homopolymer Chain, Phys. Rev. E 79, 050801(R) (2009).

[47] C. Maffi, M. Baiesi, L. Casetti, F. Piazza, and P. De Los Rios, First-Order Coil-Globule Transition Driven by Vibrational Entropy, Nat. Commun. 3, 1065 (2012).

[48] P. G. Wolynes, Folding Funnels and Energy Landscapes of Larger Proteins within the Capillarity Approximation, Proc. Natl. Acad. Sci. U.S.A. 94, 6170 (1997).

[49] J. Sabelko, J. Ervin, and M. Gruebele, Observation of Strange Kinetics in Protein Folding, Proc. Natl. Acad. Sci. U.S.A. 96, 6031 (1999).

[50] D. Sornette, Critical Phenomena in Natural Sciences: Chaos, Fractals, Self-Organization and Disorder: Concepts and Tools (Springer Science, New York, 2006).

[51] T. Chen, K. Dave, and M. Gruebele, Pressure- and HeatInduced Protein Unfolding in Bacterial Cells: Crowding vs. Sticking, FEBS Lett. 592, 1357 (2018).

[52] Y. M. Bar-On, R. Phillips, and R. Milo, The Biomass Distribution on Earth, Proc. Natl. Acad. Sci. U.S.A. 115, 6506 (2018).

[53] K. Binder, Finite Size Scaling Analysis of Ising Model Block Distribution Functions, Z. Phys. B 43, 119 (1981).

[54] K. G. Wilson, Renormalization Group and Critical Phenomena. I. Renormalization Group and the Kadanoff Scaling Picture, Phys. Rev. B 4, 3174 (1971).

[55] A. Attanasi, A. Cavagna, L. Del Castello, I. Giardina, S. Melillo, L. Parisi, O. Pohl, B. Rossaro, E. Shen, E. Silvestri, and M. Viale, Finite-Size Scaling as a Way to Probe NearCriticality in Natural Swarms, Phys. Rev. Lett. 113, 238102 (2014).

[56] S. Bradde and W. Bialek, PCA Meets RG, J. Stat. Phys. 167, 462 (2017).

[57] E. A. Guggenheim, The Principle of Corresponding States, J. Chem. Phys. 13, 253 (1945).
[58] Z. V. Djordjevic, I. Majid, H. E. Stanley, and R. Dos Santos, Correction-to-Scaling Exponents and Amplitudes for the Correlation Length of Linear Polymers in Two Dimensions, J. Phys. A 16, L519 (1983).

[59] H. J. Dyson and P. E. Wright, Intrinsically Unstructured Proteins and Their Functions, Nat. Rev. Mol. Cell Biol. 6, 197 (2005).

[60] C. Clementi, H. Nymeyer, and J. N. Onuchic, Topological and Energetic Factors: What Determines the Structural Details of the Transition State Ensemble and En-Route Intermediates for Protein Folding? An Investigation for Small Globular Proteins, J. Mol. Biol. 298, 937 (2000).

[61] P. L. Freddolino, C. B. Harrison, Y. Liu, and K. Schulten, Challenges in Protein-Folding Simulations, Nat. Phys. 6, 751 (2010).

[62] G. A. Papoian, Coarse-Grained Modeling of Biomolecules (CRC, Boca Raton, 2017).

[63] J. D. Bryngelson and P. G. Wolynes, Spin Glasses and the Statistical Mechanics of Protein Folding, Proc. Natl. Acad. Sci. U.S.A. 84, 7524 (1987).

[64] P. E. Leopold, M. Montal, and J. N. Onuchic, Protein Folding Funnels: A Kinetic Approach to the SequenceStructure Relationship, Proc. Natl. Acad. Sci. U.S.A. 89, 8721 (1992).

[65] J. Hu, T. Chen, M. Wang, H. S. Chan, and Z. Zhang, A Critical Comparison of Coarse-Grained Structure-Based Approaches and Atomic Models of Protein Folding, Phys. Chem. Chem. Phys. 19, 13629 (2017).

[66] N. Hillson, J. N. Onuchic, and A. E. García, PressureInduced Protein-Folding/Unfolding Kinetics, Proc. Natl. Acad. Sci. U.S.A. 96, 14848 (1999).

[67] H. J. Berendsen, D. van der Spoel, and R. van Drunen, GROMACS: A Message-Passing Parallel Molecular Dynamics Implementation, Comput. Phys. Commun. 91, 43 (1995).

[68] L. D. Landau, E. M. Lifshitz, and L. P. Pitaevskii, Course of Theoretical Physics: Statistical Physics Pt. 1 (Pergamon, New York, 1980). 